\title{
Shear avalanches in plastic deformation of a metallic glass composite
}

\author{
X. Tong ${ }^{1}$, G. Wang ${ }^{1}{ }^{*}$, J. Yi $^{1}$, J.L. Ren ${ }^{2}$, S. Pauly ${ }^{3}$, Y.L. Gao ${ }^{1}$, Q.J. Zhai ${ }^{1}$, N. Mattern ${ }^{3}$, \\ K.A. Dahmen ${ }^{4}$, P.K. Liaw ${ }^{5}$, J. Eckert ${ }^{6,7}$ \\ ${ }^{1}$ Laboratory for Microstructures, Shanghai University, Shanghai 200444, China \\ ${ }^{2}$ School of Mathematics and Statistics, Zhengzhou University, Zhengzhou 450001, China \\ ${ }^{3}$ IFW-dresden, Institute for Complex Materials, P.O. Box 270116, D-01069 Dresden, Germany \\ ${ }^{4}$ Department of Physics, University of Illinois at Urbana Champaign, 1110 West Green Street, \\ Urbana IL 61801, USA \\ ${ }^{5}$ Department of Materials Science and Engineering, The University of Tennessee, Knoxville, TN \\ 37996, USA \\ ${ }^{6}$ Erich Schmid Institute of Materials Science, Austrian Academy of Sciences, Jahnstraße 12, \\ A-8700 Leoben, Austria \\ ${ }^{7}$ Department Materials Physics, Montanuniversität Leoben, Jahnstraße 12, A-8700 Leoben, Austria \\ *Corresponding author: g.wang@,shu.edu.cn
}

\begin{abstract}
Changes in intermittent shear avalanches during plastic deformation of a $\mathrm{Cu}_{50} \mathrm{Zr}_{45} \mathrm{Ti}_{5}$ (atomic percent) alloy in response to variant structures including fully glassy phase and/or nanocrystal/glass binary phase are investigated. Second crystalline phases are introduced into the glassy-phase matrix of a $\mathrm{Cu}_{50} \mathrm{Zr}_{45} \mathrm{Ti}_{5}$ metallic glass to interfere with the shear-avalanche process, which can release the shear-strain concentration, and then tune the critically-dynamic behavior of the shear avalanche. By combining microstructural observations of the nanocrystals with the statistical analysis of the corresponding deformation behavior, we determine the statistic distribution of shear-avalanche sizes during plastic deformation, and established its dependence on the geometric distribution of nanocrystals. The scaling behavior of the distribution of shear-avalanche sizes follows a power-law relation accompanied by an exponentially-decaying scaling function in the pure metallic glass, and the metallic glass containing the small nanocrystals, which can be described by the mean-field theory. The large shear-avalanche events are dominated by structural tuning-parameters, i.e., the resistance of shear banding, and the size and volume fraction of the second crystalline phase in metallic glasses.
\end{abstract}

Keywords: Metallic glasses composites; Second phase; Dynamics of shear avalanche; Nanotwin 


\section{Background}

Shear avalanche is believed to be a main mechanism dominating the plastic deformation of metallic glasses at low temperatures due to the absence of crystalline defects, such as dislocations, grain boundaries, and stacking faults, etc. (Schuh et al., 2004; Klaumünzer et al., 2011a; Trexler and Thadhani, 2010; Song and Nieh, 2011; Sun et al., 2014; Cheng et al., 2009; Sun et al., 2012; Antonaglia et al., 2014b; Wang et al., 2009; Neuhaüser, 1993; Wu et al., 2011a). The shear-banding behavior is manifested in the plastic regime by repeating cycles of sudden stress drops, followed by reloading elastically, the so-called serrations or jerky flow (Wang et al., 2009). In analogy to the smooth macroscopic-scaled crystalline plasticity, which arises from the spatio-temporal averages of disruptive earthquake-like events at the nanometer scale (Richeton et al., 2005), the dynamics of serrated flows in metallic glasses have been pointed out to follow a self-organized critical (SOC) behavior (for ductile metallic glasses) or chaotic behavior (for brittle metallic glasses) according to their different plasticities (Sun et al., 2010; Sarmah et al., 2011; Liu et al., 2013b; Ren et al., 2012). Acoustic-emission experiments have clearly shown that the dynamic behavior of the shear-avalanche can be associated with the elementary mechanisms of energy dissipation (Klaumünzer et al., 2011a; Kumar and Ananthakrishna, 2011; Koslowski et al., 2004; Dimiduk et al., 2006). This energy relaxation duration is significantly affected by the environmental temperature (Klaumünzer et al., 2011b), strain rate (Liu et al., 2013a; Ren et al., 2011), and outside geometric confinement (Wang et al., 2009), which can modify the dynamic behavior of metallic glasses. From this point, a crucial question is raised: how does the dynamics of the intermittent deformation change, when this jerky flow is disturbed, and how does then the plastic deformability of metallic glasses change? Considerable experimental efforts have established that in disordered systems plasticity involves primary units, such as irreversible rearrangements of small clusters (Miracle, 2004; Schuh and Lund, 2003; Ye et al., 2010). Inspired by Eshelby transformations (Eshelby, 1957), Bulatov et al. (Bulatov and Argon, 1994) inferred that such local rearrangements should generate an elastic concordant field. Furthermore, $\mathrm{Wu}$ et al. found that the activation of shear banding was associated with the fluctuations and the irreversible changes in local strain fields (Wu et al., 2015). Thus, since each cluster alters the strain field in its surroundings, the flowing system is submitted to a self-generated dynamical noise. In metallic glasses, once this surrounding elastic-strain field approaches the yielding shear strain, the plastic flow can be activated, which is manifested to be serration events (Antonaglia et al., 2014a; Fornell et al., 2009; Huang et al., 2014).

Each serration event includes an elastic-energy accumulation and an energy-dissipation process, i.e., a shear avalanche (Klaumünzer et al., 2011a; Dimiduk et al., 2006; Liu et al., 2013a). Prohibiting the shear avalanche may enhance the toughness of metallic glasses, and promote their plasticity. One of the toughening mechanisms in bone for example suggests that deformation energy 
can be dissipated by shearing of a thin 'glue' layer between mineral-reinforced collagen fibrils at the crack tip (Peterlik et al., 2006; Gupta et al., 2005; Ritchie, 2011). Motivated by this toughening mechanism in bone, we propose that the introduction of a "plastic medium" into the glassy phase can possibly retard the shear-band propagation, and then postpone the occurrence of a shear avalanche (Fan et al., 2006). Furthermore, the plastic medium embedded into the glassy phase can interact with the shear bands (Fan et al., 2006; Wang and $\mathrm{Xu}, 2013$; $\mathrm{Xu}$ and Ma, 2014). Local interactions can give rise to a strain accommodation (Fan et al., 2006). The local stress also resultantly increases. Their occurrence and properties are believed to largely influence the macroscopic behavior of metallic glasses. Therefore, the exploration of the interaction between the second phase and shear bands is expected to provide the valuable structural information for the improvement of the plasticity of metallic glasses.

Recent research found that twins can form in a nanocrystals-embedded $\mathrm{Cu}-\mathrm{Zr}$ binary glassy matrix (Pauly et al., 2010a; Wu et al., 2010). The B2 phase as a second phase can be observed in CuZr-based metallic-glass composites under tensile deformation, which induces a macroscopically-detectable plastic strain and significant work hardening (Wu et al., 2011b; Pauly et al., 2010b). The size scale, the distribution, as well as the mechanical properties of the second phase determine the properties of the metallic-glass composites (Pauly et al., 2009), which can effectively prevent the catastrophic failure in brittle metallic glasses. Accordingly, we are interested in the question whether the serrations in the plastic flow can be changed by the precipitation of the second crystalline phase.

In this paper, we choose a $\mathrm{Cu}_{50} \mathrm{Zr}_{45} \mathrm{Ti}_{5}$ (atomic percent, at.\%) alloy as a model material to investigate the influence of the local structure on the dynamics of shear avalanches. $\mathrm{The} \mathrm{Cu}_{50} \mathrm{Zr}_{45} \mathrm{Ti}_{5}$ alloy has a significant compressive plastic strain (Wang et al., 2014), which facilitates to analyze the serration events. Furthermore, the $\mathrm{Cu}_{50} \mathrm{Zr}_{45} \mathrm{Ti}_{5}$ alloy exhibits a relative low glassy-forming ability, compared with Zr-based metallic glasses, indicating that the structure of the glassy-phase matrix is sensitive to the cooling rate. Through carefully-controlling the cooling rate, nanocrystals with different sizes and volume fractions can precipitate in the glassy-phase matrix. Then, we expect that the different crystalline defects, such as twinning, can be introduced into these nanocrystals, and further affect the dynamics of the serrated flow behavior.

\section{Experimental procedure}

Alloy ingots were prepared by arc-melting a mixture of pure metal elements [with the purity higher than $99.99 \%$ (weight percent, wt.\%)] in a titanium-gettered argon atmosphere, followed by suction casting into copper molds to form cylindrical samples with dimensions of $\Phi 2 \times 70 \mathrm{~mm}$. Fractography was observed using a HITACH TM-1000 scanning electron microscope (SEM). 
X-ray diffraction (XRD) measurements were performed, employing a Siemens microdiffractometer with the $\mathrm{Cu}-\mathrm{K}_{\alpha}$ radiation $(\lambda=0.1542 \mathrm{~nm})$. The operation voltage and current were $40 \mathrm{kV}$ and 150 $\mathrm{mA}$, respectively. The measured angle was from $20^{\circ}$ to $63^{\circ}$. High-resolution transmission electron microscopy (HRTEM) was conducted in a JEOL JEM-2010F microscope operated at an accelerating voltage of $200 \mathrm{kV}$. The transmission electron microscopy (TEM) samples were prepared by polishing and subsequent ion-milling on a Gatan 691 Precision Ion Polishing System with a liquid-nitrogen cooling system. The ion energy of the ion beam and beam angle were 2.5 $\mathrm{keV}$ and $5^{\circ}$, respectively. The ion-milling durations for all of the samples were $2.5 \sim 3 \mathrm{~h}$. Compressive test specimens were fabricated from the as-cast samples by means of a diamond saw with cooling water. They had a height of $4 \mathrm{~mm}$ and a diameter of $2 \mathrm{~mm}$. Compressive tests were conducted, using an Instron 8562 machine at a strain rate of $2.5 \times 10^{-4} \mathrm{~s}^{-1}$.

\section{Results}

The cooling rate in the chamber of the copper mold is not homogeneous. The sensitivity of the $\mathrm{Cu}_{50} \mathrm{Zr}_{45} \mathrm{Ti}_{5}$ alloy to the cooling rate can generate different microstructures. Figure 1 summarizes the stress-strain behavior, and the XRD patterns of four compressive samples cut from different positions of the as-cast rod (Wang et al., 2014). The four samples are named PT I, PT II, PT III, and PT IV, respectively. PT I corresponds to the sample firstly solidified, which is located at the bottom of the rod. With increasing the position from the bottom to the top of the rod-like sample, PT II, PT III, and PT IV were cut. PT IV is solidified at the last time, located at the top of the rod-like sample. These four samples exhibit different stress-strain behaviors depending on their structures. To exclude the occasional cases, the compression tests of each sample are repeated three times, which are plotted in Fig. S1 in Supplementary Materials (see Supplemental materials). In the present study, PT I, PT II, PT III, and PT IV are cut from the same rod-like sample because it is reasonable to construct the relation between the microstructures, and the mechanical behavior of the $\mathrm{Cu}_{50} \mathrm{Zr}_{45} \mathrm{Ti}_{5}$ alloy. PT I exhibits elastic deformation, followed by the serrated-plastic flows. The corresponding structure of PT I is fully glassy, i.e., a broad diffraction peak shown on the XRD pattern (see the inset of Fig. 1). PT II also shows the elasto-plastic deformation behavior, which is similar to that in PT I. However, the plastic strain of PT II is slightly larger than that of PT I, and the yield strength and elastic modulus are decreased, as compared to those of PT I. The XRD pattern of PT II shows that some tiny peaks overlap on the broad diffraction peak, which can be attributed to the precipitation of nanocrystals. For PT III and PT IV, they exhibit a decreased yield strength, a decreased elastic modulus, and an increased plasticity, as compared to PT I. Especially, PT IV displays a significant strain-hardening behavior, and a large plastic strain of 0.113 . The plastic-strain regime of PT IV can be divided into two parts. The first part, which is in the strain range from 0.019 to 0.102 , is 
composed of numerous small serration events that are corresponding to the shear-banding operation. The second part, which is in the range from 0.102 to the fracture, exhibits several large serration events, which is highlighted by a dash-point rectangle in Fig. 1. These large serration events usually can lead to the formation of shear steps, which then causes obvious shear slip on the fracture surface (Jiang and Atzmon, 2003; Georgarakis et al., 2008; Qiao et al., 2012). The mechanical-properties parameters are summarized in Table I. The XRD patterns of PT III and PT IV show relatively-pronounced crystalline peaks (see the inset of Fig. 1). The calculation of $d$ spacings obtained from the most probable peaks points to three main phases: the CuZr cubic B2 phase $(\mathrm{a}=3.256 \AA)$, the $\mathrm{CuZr} \mathrm{B19}$ ' phase $(\mathrm{a}=3.301 \AA, \mathrm{b}=4.138 \AA)$ and the $\mathrm{Cu}_{10} \mathrm{Zr}_{7}$ orthorhombic phase ( $\mathrm{a}=12.675 \AA, \mathrm{b}=9.313 \AA$, and $\mathrm{c}=9.347 \AA$ ), which are marked by circles and squares, respectively. The XRD patterns of PT II, PT III, and PT IV show that the crystalline peaks become sharper and sharper, which implies that the size of the crystalline phases increases, based on the Scherrer formula (Cullity, 1959). It is evident that the precipitation of nanocrystals decreases the yield strength, and the elastic modulus of the $\mathrm{Cu}_{50} \mathrm{Zr}_{45} \mathrm{Ti}_{5}$ alloy (Peterlik et al., 2006).

After the compression tests, all fractured samples were observed by SEM. It can be seen that the fracture angles, i.e., the angle between the fracture surface and the loading direction, are constant at a value of $43^{\circ}$, and numerous shear bands are formed on the surfaces (see Fig. 2). The fractographic analysis of PT I, PT II, and PT III reveals vein-like patterns, which suggests that a viscous layer formed on the fracture surface due to adiabatic heating, and shear dilatation [see Figs. 2(a), (c), and (e)] (Lewandowski and Greer, 2006). The fractography of PT IV shows a mixed morphology [see Fig. 2(g)], composed of a vein-like pattern and a slurry-like pattern, which indicates that the deformation mechanisms of PT IV are different from those in PT I, PT II, and PT III. Also, the lateral surface morphologies show that with increasing the plastic strain, the density of shear bands increases [see Figs. 2(b), (d), (f), and (h)].

The structures of all four fractured samples were further observed by HRTEM to explore the mechanism of plastic flows. In Fig. 3(a), the high-resolution image of PT I exhibits a mazy and homogeneous contrast pattern, which is typical of a monolithic glassy phase. Figure 3(b) shows a dark-field image of PT I, which exhibits a homogeneous contrast. The inset of Fig. 3(b) shows a selected area electron diffraction (SAED) pattern with two diffraction halos. All these features present an inherent glassy character of the fractured PT I. The HRTEM image of PT II shows some lattice fringes that are marked by white circles [see Fig. 3(c)]. Correspondingly, some white spots fragmentarily distribute on the dark-field image [see Fig. 3(d)], which illustrates that some crystalline phases precipitate in the glassy-phase matrix. Due to the small volume fraction and the small size of the crystalline phases in PT II, the corresponding SAED pattern in the inset of Fig. 3(d) also shows two main bright diffraction halos, which is similar to that in PT I. For PT III, the 
size of the lattice fringe patterns is larger than those in PT II [see Fig. 3(e)]. The corresponding dark-field image indicates some significantly more white areas, compared to those shown in PT II [see Fig. 3(f)]. From the SAED pattern exhibited in the inset of Fig. 3(f), it is difficult to discern the crystalline phases. The high-resolution image clearly suggests that the deformed PT IV contains some twins, as shown in Fig. 3(g). Twins are not observed in other samples. The size of the crystalline phases in PT IV is larger than those in PT II and PT III. The SAED pattern [see the inset of Fig. 3(h)] cannot provide the useful information for the crystalline phase structure. The dark-field image of PT IV shows that many white areas corresponding to the crystalline particles are visible, which suggests that the volume fraction of the crystalline phases in PT IV is larger than those in PT II and PT III. The average diameters of the crystalline phase, $D_{a v r}$, of PT II, PT III, and PT IV are measured to be $8.3 \pm 0.9 \mathrm{~nm}, 11.1 \pm 1.8 \mathrm{~nm}$, and $20.2 \pm 2.8 \mathrm{~nm}$, respectively. Through measuring the areas of white spots in the dark-field images, the volume fractions of crystalline phases, $f_{c}$, of PT II, PT III, and PT IV are estimated to be $7.8 \pm 1.0 \%, 16.8 \pm 1.9 \%$, and $35.0 \pm 2.9 \%$, respectively. The calculation of the volume fraction of the crystalline phase is described in Fig. S4 in Supplementary Materials (see Supplemental materials). The structural information deduced from the TEM results are also listed in Table II. Previous studies have found that the plastic deformation could activate the crystallization in the glassy phase, or at least could enhance the crystallization process (Chen et al., 2006). For comparison, the TEM images of the alloys before deformation are shown in Fig. S5 in Supplementary Materials (see Supplemental materials). The structural parameters of the four undeformed alloys are listed in Table SI in Supplementary Materials (see Supplemental materials). Although the volume fractions and the sizes of the nanocrystals in PT II, PT III, and PT IV before the deformation are slightly smaller than those deformed samples, the differences are too small to be meaningful. Thus, it can be reasonably supposed that the plastic deformation does not significantly change the microstructures in the $\mathrm{Cu}_{50} \mathrm{Zr}_{45} \mathrm{Ti}_{5}$ alloys.

The magnified stress-strain curves in the plastic regimes clearly show serrations corresponding to the generation, spreading, and termination of shear bands (see Fig. 4) (Wang et al., 2009; Ren et al., 2011). One serration event includes a process of elastic-energy accumulation and a process of elastic-energy release. The elastic-energy density covered by one serration event, $\Delta \delta$, can be expressed as,

$\Delta \delta=\left(\Delta \sigma^{\prime} \Delta \varepsilon^{\prime}\right) / 2$,

where $\Delta \sigma^{\prime}$ and $\Delta \varepsilon^{\prime}$ are the elastic stress and strain in the serration event, respectively, which are indicated in Fig. 4. In the elastic energy release process, the stress drop, $\Delta \sigma$, in the serration event reflects the elastic energy stored in the sample released by a shear avalanche (see Fig. 4) (Cheng et al., 2009; Sun et al., 2010; Ren et al., 2014; Jiang et al., 2008). The release of the elastic energy causes adiabatic heating and dilatation of the glassy phase in the shear band (Lewandowski and 
Greer, 2006; Batra and Lear, 2005), which significantly decreases the viscosity of the glassy phase. Then, a grease-like layer is formed in the shear band. As such, the distance of shear-band propagation, i.e., the shear-avalanche size, can be inspected from the value of the elastic-energy density stored in each serration event. The elastic-energy density as a function of the true strain is plotted in Fig. 5, which indicates that the distribution of the shear-avalanche size is stochastic in the temporal space. On the other hand, the shear-band formation places, and the formation times in the $\mathrm{Cu}_{50} \mathrm{Zr}_{45} \mathrm{Ti}_{5}$ alloy cannot be predicted (see Fig. 2), suggesting that the spatial distribution of the shear avalanche is stochastic. Therefore, the statistical analysis of the shear-avalanche size is employed to further characterize the shear avalanche (Wang et al., 2009; Sun et al., 2010).

After ergodically processing the elastic-energy density, $\Delta \delta$, the cumulative distributions of the $\Delta \delta$ value, i.e., the percentage of the number of the elastic-energy density larger than a given value, $C(>\Delta \delta)$, of four alloys are shown in Fig. 6. Two stages in the distributions of PT I, PT II, and PT III are recognized. Numerous smaller shear avalanches, which follow a power-law distribution, constitute Stage I. Stage II is composed of several larger shear avalanches, in which the distribution of these shear avalanches exhibits a fast decay. The cumulative distributions (consisting of Stages I and II) of the elastic-energy density can be predicted by a power-law distribution function (Antonaglia et al., 2014b),

$C(\Delta \delta) \sim \Delta \delta^{-(\kappa-1)} F\left(\Delta \delta / \Delta \delta_{c}\right)$,

where $F\left(\Delta \delta / \Delta \delta_{c}\right)\left(=\exp \left[-\left(\Delta \delta / \Delta \delta_{c}\right)^{2}\right]\right)$ is a quickly-decaying scaling function (Csikor et al., 2007), $\kappa$ is a scaling exponent, and $\Delta \delta_{c}$ is the size of the largest "critical" avalanche that acts as a cut-off in $F\left(\Delta \delta / \Delta \delta_{c}\right)$, and decays exponentially for $\Delta \delta / \Delta \delta_{c} \geq 1$. To obtain the $\kappa$ and $\Delta \delta_{c}$ values, Equation (2) is used to fit the experimental results plotted in Fig. 6 . The $\kappa$ and $\Delta \delta_{c}$ values are the variables. The fitting process was carried out in the softwares of Matlab and Origin. After giving the initial values of $\kappa$ and $\Delta \delta_{c}$, the iteration program can run until the adjustable $R$-square value, $R^{2}$, approaching 0.97 . After that, the iteration program is stopped, and generates the fitting results including the $\kappa$ and $\Delta \delta_{c}$ values, which are listed in Fig. 6. The parameters of $\kappa$ and $\Delta \delta_{c}$ reflect the profile of the shear-avalanche size distribution, which can characterize the deformation mechanism, and the characteristic shear avalanche in metallic glasses, respectively (Richeton et al., 2005). The $\Delta \delta_{c}$ value is marked by green-dash lines in Fig. 6, which is the deviation point between the power-law behavior (Stage I), and the exponential decay behavior (Stage II). As shown in Fig. 6, the average $\kappa$ values of PT I, PT II, and PT III are $1.5 \pm 0.1$, suggesting that the inhomogeneous deformations of these three alloys are not influenced by the observed structural differences. The exponent of 1.5 is in excellent agreement with the mean-field theory (MFT) (Dahmen et al., 2009; Ben-Zion et al., 2011). With increasing the shear-avalanche size to the "critical" value, i.e., the cut-off value, $\Delta \delta_{c}$, the rapidly-decaying term in Eq. (2) comes to work. The cut-off values of $\Delta \delta_{c}$ increase from 
approximately $1,205 \pm 103 \mathrm{~J} / \mathrm{m}^{3}$ to $6,062 \pm 373 \mathrm{~J} / \mathrm{m}^{3}$ with increasing the size and the volume fraction of nanocrystals, which indicates that the large nanocrystals can effectively influence the shear-banding behavior. However, further enlarging the nanocrystals causes that the cumulative distribution of the shear-avalanche size of PT IV exhibits a bimodal case. It can be seen that a broken slope in the power-law regime: an exponent, $\kappa=2.0 \pm 0.1$, is recovered at small shear-avalanche sizes, whereas $\kappa=1.26 \pm 0.03$ is observed at larger scales (see Fig. 6). The cut-off value, $\Delta \delta_{c}$, of PT IV increases to approximately $14,583 \pm 950 \mathrm{~J} / \mathrm{m}^{3}$. As a characteristic shear-avalanche size, the $\Delta \delta_{c}$ value can reflect the shear avalanche occurring on the large scale (Csikor et al., 2007), and is associated with the structure of materials (Richeton et al., 2005). The repeated compression tests of four alloys, which are plotted in Fig. S1, are also carried out the statistical analysis of the serration events. Figure S2 in Supplementary Materials (see Supplementary materials) suggests that the values of $\kappa$ and $\Delta \delta_{c}$ from four alloys are almost consistent with the results in Fig. 6, which excludes the occasional results.

\section{Discussion}

Previous studies have found that scale-free dislocation avalanches could be observed in single crystals, and grain boundaries acted as barriers for the dynamic propagation of avalanches (Richeton et al., 2005; Dimiduk et al., 2006; Csikor et al., 2007). In the $\mathrm{Cu}_{50} \mathrm{Zr}_{45} \mathrm{Ti}_{5}$ alloy, the structural medium for delivering the plastic strain is shear bands rather than dislocations, and the critical shear-avalanche behavior in PT I, PT II, and PT III can be described by the MFT (Antonaglia et al., 2014a, b; Dahmen et al., 2011). Thus, the structural media delivering the plastic strain are elastically coupled. For metallic glasses, it means that a sliding shear band can trigger the shear avalanches of other shear bands (Chen et al., 2013). This process requires addressing the spatial interaction between neighboring shear banding during plastic deformation. On the other hand, the nanocrystals temporally interact with the shear bands, which leads us to further explore the local shear-strain rate in each serration event.

In the present study, the stress (or strain) drop in each serration event (see Fig. 4) could be simplified as a process that the elastic energy is released to generate one shear band. Thus, the shear stress, $\tau_{s}$, activating a shear band can be expressed as (Smith and Hashemi, 2006; Song and Nieh, 2011),

$\tau_{s}=\sigma_{p} \sin \theta \cos \theta$

where $\theta$ is the fracture angle $\left(43^{\circ}\right)$ measured from Fig. 2, and $\sigma_{p}$ is the peak stress in the serration event, as marked in Fig. 4. The shear stress in each serration as a function of plastic strain for four alloys are plotted in Fig. 7(a), according to which an increasing trend of the shear stress in each serration event is observed with increasing plastic strain. Correspondingly, the strain drop, $\Delta \varepsilon$ (see 
Fig. 4), consists of the shear component, $\gamma$, and the normal component, $\varepsilon_{\mathrm{n}}$, as shown in Fig. 8. The shear strain, $\gamma$, can be calculated by $\gamma=(\Delta \varepsilon H) /(h \cos \theta)$, where $H$ is the height of the compressive sample, and $h$ is the thickness of shear bands (The deduction of the equation for calculation of the $\gamma$ value is documented in Supplementary Materials.). Here, the thickness of the shear band is approximately $10 \mathrm{~nm}$ (Zhang and Greer, 2006; Wang et al., 2006; Bailey et al., 2006; Zhao et al., 2013). Moreover, from the strain-time curves (not shown), the durations of the strain drops can be measured. Therefore, the shear-strain rates, $\dot{\gamma}$, corresponding to each serration event in four samples are plotted in Fig. 7(b). It is evident that most of the shear-strain rates of four alloys are in the range of $10^{1}$ to $10^{3} \mathrm{~s}^{-1}$. The average shear-strain rates of four alloys are approximately $\sim 10^{2} \mathrm{~s}^{-1}$ [see Fig. 7(b)], which decreases with increasing the size and the volume fraction of the crystalline phase. In other words, the precipitation of nanocrystals retards the shear avalanches. At the slow shear-strain rate, a shear avalanche finishes before the next one is started, suggesting that the elastic interaction between the neighboring shear bands is effectively activated in the temporal space, and also sufficiently long-ranged. Thus, the MFT, which assumes infinite range interactions, can correctly predict the scaling behavior of the shear-avalanche statistics on large length scales (Antonaglia et al., 2014b). Vice versa, at the fast shear-strain rates, the elastic interaction between shear avalanches is not significant in the temporal space, which means that the MFT may not be valid. Once the shear band is formed, an elastic-strain field can appear in the front of the shear-band tip, which is analogy to the elastic-strain field in the front of the crack tip. The size of the elastic-strain field is several times larger than the inter-spacing between two neighboring shear bands (Liu et al., 2013a), which causes the overlap of two elastic-strain fields initiated from neighboring shear bands. The overlap of the elastic-strain field can result in a hierarchy of length scales (Bharathi et al., 2001), leading to a self-organized critical behavior that is manifested as a scaling behavior of the shear-avalanche statistics. The nanocrystal/glass interfaces, as a kind of defect, can initiate more shear bands after yielding as compared to the case of PT I (Pauly et al., 2010b). The density of shear bands increasing can enhance the plastic deformation ability of the alloy, and decrease the inter-space between two neighboring shear bands. Thus, the spatial interaction of the neighboring elastic-strain fields is enhanced, in which the scaling behavior, i.e., the power-law regime, of the shear-avalanche statistic is more significant, as shown in the cases of PT II, PT III and PT IV (see Fig. 4).

When the size of nanocrystals is further increased, nanocrystals can accommodate the shear deformation by twinning. The deformation mechanism is composed of shear banding and nano-twinning [c.f., Figs. 2(g) and $3(\mathrm{~g})$ ]. For the cumulative probability of the elastic-energy distribution, we observe a broken slope in the power-law regime, i.e., an exponent of $\kappa=2.0 \pm 0.1$ is recovered in the small shear avalanche size regime, whereas $\kappa=1.26 \pm 0.03$ is observed in the 
larger scales (see Fig. 6). The broken point is the critical point between the small shear avalanches and the large shear avalanches. On the small shear avalanche regime, the collective shear-banding behavior self-organizes into a scale-free pattern of shear avalanches characterized by a power-law distribution of shear-avalanche sizes (Liu et al., 2013b; Bharathi et al., 2001). On the large shear avalanche regime, the distribution also follows Eq. (2) with the exponent of $\kappa=1.26 \pm 0.03$ and the cut-off value of $\Delta \delta_{\mathrm{c}}=14,583 \pm 950 \mathrm{~J} / \mathrm{m}^{3}$. Thus, in PT IV containing larger nanocrystals, the shear-avalanche behavior does not well follow the MFT. As compared to the shear bands formed in the earlier plastic-deformation stage, the numbers of shear steps, corresponding to the large serration events, are less. In this case, the elastic interaction due to the shear-step formation is not significant. The collective shear-banding behavior is absent, which indicates that the MFT cannot be effective in the large scale serration events.

Besides the exponent of $\kappa$, Equation (2) introduces the squared exponential decay function into the power-law relation accounts for the finite elastic energy in the serration event (Freltoft et al., 1986). The cut-off value, $\Delta \delta_{c}$, reflects the shear-avalanche behavior on large scales (Zaiser and Moretti, 2005), and plays an important role in the rupture process of materials (Richeton et al., 2005; Zaiser and Moretti, 2005; Papanikolaou et al., 2012). In crystalline materials, the cut-off value is found to be associated with the strength of the deformation medium (Antonaglia et al., 2014b; Dahmen et al., 2011), and/or the geometric dimension of the crystalline grains (Richeton et al., 2005), which leads us to focus on the factors tuning the cut-off value.

For the monolithic metallic glass, it is well known that shear bands preferentially originate from some weak spots, when the external stress approaches a critical value, i.e., yield stress. These weak spots usually exhibit a relative low strength, which are considered to be the loosely-bonded atomic clusters (Ding et al., 2014; Yang et al., 2005). With increasing the stress, these weak spots (shear transformation zones, STZs) can aggregate to form shear bands, and then cause a dilatation of the glassy phase in the shear bands, which decreases the viscosity of the glassy phase. Simultaneously, adiabatic heating also enhances the decrease in viscosity (Lewandowski and Greer, 2006). Thus, the shear band in the metallic glass can be treated as a weak zone, along which the shear avalanche occurs (Lewandowski and Greer, 2006; Yang et al., 2005). The shear viscosity, $\eta$, of the glassy phase in the shear band is associated with the shear stress, $\tau_{s}$, and the shear-strain rate, $\dot{\gamma}$, i.e. (Song and Nieh, 2011; Song and Nieh, 2009),

$\eta=\frac{\tau_{s}}{\dot{\gamma}}$

According to Eqs. (3) and (4), Figures 7(a) and (b), the shear viscosity of the glassy phase in the shear band can be estimated in Fig. 7(c). It can be seen that the shear viscosities of PT I, PT II, and PT III fluctuate around $10^{6} \mathrm{~Pa} \cdot \mathrm{s}$. For PT IV, the shear viscosity increases significantly, which is in 
the range of $10^{6}$ to $10^{8} \mathrm{~Pa} \cdot \mathrm{s}$. The average shear viscosities of the four alloys are listed in Fig. 7(c). For the metallic glass (PT I), the shear viscosity is approximately $1.42 \times 10^{6} \mathrm{~Pa} \cdot \mathrm{s}$, which is consistent with the value reported in Ref. (Song and Nieh, 2011).

In PT II, some nanocrystals with the average diameter of $8.3 \pm 0.9 \mathrm{~nm}$ precipitate into the glassy-phase matrix, which is smaller than the typical thickness of shear bands. These nanocrystals might "float" in the viscous glassy phase in shear bands because the nanocrystals cannot act as the medium to deliver the plastic strain or stop the shear slipping, as shown in Fig. 8(b). Therefore, the mechanical behavior, such as the yield strength of PT II, is slightly changed in PT II due to the nanocrystals. The nanocrystals actually are considered as "hard" particles that can slightly increase the shear viscosity to $1.48 \times 10^{6} \mathrm{~Pa} \cdot \mathrm{s}$, which has been well documented previously (McLelland, et al., 1997; Kirkwood, 1994). Accordingly, the resistance to shear sliding is improved, which means that localized shear banding, i.e., the shear avalanche, of PT II is more difficult to be activated as compared to that in PT I. As such, more elastic energy is required to slide the shear bands in the serration events as compared to the case in PT I, which can shorten the shear-sliding distance (see Fig. 4). Then, the critical shear avalanche, i.e., the $\Delta \delta_{\mathrm{c}}$ value, is pushed to be a higher value.

The average size of the nanocrystals in PT III is increased to be approximately $11.1 \pm 1.8 \mathrm{~nm}$ that already can be compared to the thickness of shear bands. With elastically increasing the external stress, a jam of shear strains (stresses) can be formed at the interface between the nanocrystalline phase and the glassy phase. When the stress-concentration built-up at the interface can approach the critical yield strength of the glassy phase, the shear bands commence to slip across the nanocrystal/glass interface. In this way, new plastic slip can originate from the interface, which results in a significant decrease in the yield strength of PT III, and more shear-band initiation, confirmed by the stress-strain curve (c.f., Fig. 1) and the SEM image [c.f., Fig. 2(f)]. When a running shear band encounters the nanocrystals, slipping can be prohibited, as shown in Fig. 8(c), which is manifested as a slowdown in the shear-strain rate, and an increase in the shear viscosity correspondingly $\left(1.53 \times 10^{6} \mathrm{~Pa} \cdot \mathrm{s}\right)$ (see Fig. 7). The shear-sliding distance is resultantly decreased, causing a reduction in the amplitude of serration events, i.e., the shear-avalanche size. On the other hand, due to the prohibition of the nanocrystals, the shear fracture is postponed. Therefore, the critical shear avalanche, $\Delta \delta_{\mathrm{c}}$, is increased to $6,062 \pm 373 \mathrm{~J} / \mathrm{m}^{3}$.

For PT IV, nano-twinning occurs in the glassy-phase matrix, which possibly causes that the yield shear strength of PT IV to decrease to $793 \pm 61 \mathrm{MPa}$ according to Eq. (3) and Table I. For the monolithic $\mathrm{Cu}_{50} \mathrm{Zr}_{45} \mathrm{Ti}_{5}$ metallic glass, the yield shear stress is $868 \pm 23 \mathrm{MPa}$, which can be roughly treated as the critical shear strength for the shear-band formation in the glassy phase. Thus, when the yield strength is approached in PT IV, the plastic deformation should occur preferentially around the nanocrystals. The elastic stress locally accommodates at the interface between the 
nanocrystal and the glass phases. A stress gradient can develop at the center of the nanocrystals (Lund and Schuh, 2007; Miller and Acharya, 2004), which can be enlarged to be $\sim 2$ - 3 times of the stress accommodation at the interface (Lund and Schuh, 2007). The central stress in the nanocrystals reaches approximately 1,586 - 2,379 $\mathrm{MPa}$. The formation of twins in nanocrystals is the result of the competition between the critical shear stress needed to nucleate a perfect dislocation, $\tau_{N}$, and the critical shear stress required to initiate the Shockley partial twinning dislocation to generate stacking faults and deformation twins, $\tau_{P}$ (Chen et al., 2003). According to the classical dislocation theory, $\tau_{N}$ and $\tau_{P}$ can be expressed (Chen et al., 2003),

$\tau_{N}=\frac{2 \alpha G b_{N}}{D_{a v r}}$

$\tau_{P}=\frac{2 \alpha G b_{P}}{D_{\text {avr }}}+\frac{\omega}{b_{P}}$

where $\alpha$ is a parameter reflecting the character of the dislocation, i.e., edge dislocation $(\alpha=0.5)$, and screw dislocation ( $\alpha=1.5$ ), respectively (Hirth and Lothe, 1982); $G$ is the shear modulus; $b_{N}$ and $b_{P}$ are the magnitudes of the Burgers vectors of the perfect dislocation and the partial dislocation; and $\omega$ is the stacking-fault energy. These two shear stresses are correlated to the crystal size, $D_{a v r}$. Once the $D_{a v r}$ value approaches a critical size, twinning operates, which can be expressed as (Chen et al., 2003):

$D_{\text {avr }}=\frac{2 \alpha G b_{p}\left(b_{N}-b_{p}\right)}{\omega}$.

For the crystalline phase, the $G$ value is approximately $32 \mathrm{GPa}$ (Chen et al., 2003). The $\alpha$ value is 1.0 (Chen et al., 2003). The $b_{P}$ value is approximately $0.095 \mathrm{~nm}$ (Pauly et al., 2010b). The minimum critical crystal size for the twin formation is around $15 \mathrm{~nm}$ (Pauly et al., 2010b). The $\omega$ value is $0.04 \mathrm{~J} / \mathrm{m}^{2}$ (Pauly et al., 2010b). Based on these values, the $b_{N}$ value is estimated to be 0.391 $\mathrm{nm}$ according to Eq. (7). Then, $\tau_{N}$ and $\tau_{P}$ are calculated to be $828 \mathrm{MPa}$ and $826 \mathrm{MPa}$, respectively, which are equivalent with each other, and significantly lower than the stress gradient of 1,586 2,379 $\mathrm{MPa}$ at the center of the nanocrystals. Our TEM results clearly show that nano-twinning can only occur in PT IV because the average nanocrystal size of $20.2 \pm 2.8 \mathrm{~nm}$ is larger than $15 \mathrm{~nm}$. The formation of nano-twinning can distort the strain field, and then initiate new shear bands from the interface of the nanocrystal/glass phase. Therefore, nanocrystals act as a "softer" matter to buffer the shear avalanche, which can consume the partial elastic energy by twinning, as shown in Fig. $8(\mathrm{~d})$. Then, the amplitude of the serration events is reduced, and the shear viscosity in the shear band increases to be $5.23 \times 10^{6} \mathrm{~Pa}$.s. As such, the value for the critical shear avalanche size, $\Delta \delta_{\mathrm{c}}$, is enhanced to be $14,583 \pm 950 \mathrm{~J} / \mathrm{m}^{3}$. The relationship between the $\Delta \delta_{\mathrm{c}}$ value and the shear viscosity is plotted in Fig. 9. It is obvious that the high shear viscosity, i.e., the large shear resistance, causes the larger critical shear avalanche size. 
To further characterize the spatial influences of the nanocrystals on the shear avalanches, the geometric distribution of the nanocrystals should be considered. Besides the average diameter of nanocrystals, $D_{a v r}$, the volume fraction of nanocrystals, $f_{c}$, and the average interparticle spacing, $L$, is another parameter characterizing the distribution of the nanocrystals. The $L$ value can be calculated using an equation (Pauly et al., 2009; Wang et al., 2014),

$L=D_{a v r} \sqrt[3]{\frac{\pi}{6 f_{c}}}$.

The calculated $L$ values for PT II, PT III, and PT IV are listed in Table II. Furthermore, the gaps between neighboring nanocrystals, $s$, measured from the TEM images are listed in Table II. Theoretically, the $s$ value can be obtained by $s \approx L-D_{\text {avr }}$. The $s$ value are consistent with those from the calculated values. The gap between neighboring nanocrystals is an important factor affecting the $\Delta \delta_{\mathrm{c}}$ value. For the fully glassy phase, such as PT I, the metallic glass can be treated as a composite composed of the nanocrystals with a size of $0 \mathrm{~nm}$ and the glassy phase. In this case, once a shear band is formed, the gap between neighboring nanocrystals must be larger than the width of the shear bands $(10 \mathrm{~nm})$. In Fig. 10, the $s$ value of PT I is chosen as the smallest value of $10 \mathrm{~nm}$ to construct the relationship between the $\Delta \delta_{\mathrm{c}}$ value and the $s$ value. Figure 10 plots the $\Delta \delta_{\mathrm{c}}$ value as a function of the corresponding $s$ value. It clearly shows a power-law relationship, i.e.,

$\Delta \delta_{c} \propto s^{-1.64 \pm 0.17}$.

Since $s \approx L-D_{\text {avr }}$, Equation (9) can be rewritten as,

$\Delta \delta_{c} \propto\left[D_{\text {avr }}\left(\sqrt[3]{\frac{\pi}{6 f_{c}}}\right)-1\right]^{-1.64 \pm 0.17}$.

It is obvious that a scaling relationship of $\Delta \delta_{\mathrm{c}} \propto s^{-1.64}$ can be found, and the shear avalanche in the $\mathrm{Cu}_{50} \mathrm{Zr}_{45} \mathrm{Ti}_{5}$ nanocrystal/glass phase composite is associated with the diameter and the volume fraction of the nanocrystals. There is a critical volume fraction, i.e., $f_{\mathrm{c}}=52 \%$, at which the $s$ value decreases to zero. In this case, most of the nanocrystals impinge upon each other. The deformation should, then, be dominated by the crystalline defects. The scaling property of the $\Delta \delta_{\mathrm{c}}$ value can be motivated as follows: Large shear avalanches extend along a shear plane across the entire specimen's cross-section. The total strain produced by such a "system-spanning" avalanche is reversely proportional to the interspacing between two neighboring nanocrystals, which is determined by the size and the volume fraction of the nanocrystals. It needs to point out that, although the power-law relationship between the $\Delta \delta_{\mathrm{c}}$ value and the $s$ value is explored in the present study, the accurately quantitative description of this relationship is still under investigation.

The above discussion clearly shows that the intrinsic property of the shear band (the shear viscosity) and the external confinement (the size and distribution of nanocrystals) can significantly influence the dynamic shear avalanche in metallic glasses. The nanocrystals act as barriers to the 
shear avalanches. Although we find that the increase in the shear viscosity of the shear bands can slow down the dynamic process of shear banding (see Fig. 9), the further quantitative description of the relationship between the avalanche size and the shear viscosity is under investigation. On the other hand, regarding the geometric confinement from the nanocrystals, the size of nanocrystal, $D_{a v r}$, and the volume fraction of nanocrystal, $f_{c}$, can be involved in the avalanche, as shown in Eq. (10). The larger nanocrystals effectively improve the shear avalanche size, indicating that the larger the nanocrystalline size, the quicker the avalanches are damped. Especially, larger nanocrystals not only act as barriers to the dynamic propagation of shear avalanches but also activate more small shear avalanches (such as in PT III) or transmit internal shear strain to tune the critical dynamics (such as in PT IV).

\section{Conclusions}

Through introducing nanocrystals into the glassy phase, the plasticity of the $\mathrm{Cu}_{50} \mathrm{Zr}_{45} \mathrm{Ti}_{5}$ metallic glass is improved, and the yield strength is decreased. Correspondingly, the nature of the shear avalanches in the plastic-strain regime is changed. Statistical analyses explicitly show that the universality of the scale-free intermittent flow can be applied to metallic glasses, and the nanocrystal/glass phase composite. The power-law distributions of the shear-avalanche size occur in the range covering the small shear-avalanche size, which gives evidence that the serrated flow dynamics is a self-organized critical system. Although the small nanocrystals (in size and volume fraction) do not significantly influence the deformation mechanism, i.e., shear banding, they can postpone the emergence of the large shear avalanches, and, thus, enhance the ductility. Resultantly, the scale-free pattern, i.e., the power-law regime, in the shear-avalanche-size distribution is enhanced. The MFT is used to describe the shear-banding behavior of the $\mathrm{Cu}_{50} \mathrm{Zr}_{45} \mathrm{Ti}_{5}$ composite, when the nanocrystals are small in the size and the volume fraction. For the large nanocrystals embedded into the glassy phase, twinning can occur to cooperate with shear banding. The participation of the crystalline defects in the shear-banding behavior of the glassy phase causes the dynamics of the shear avalanches to deviate from the MFT. The large shear avalanches depend on the geometric distribution of the nanocrystals. The critical shear-avalanche value scales with the gap between the neighboring nanocrystals. The plasticity of the $\mathrm{Cu}_{50} \mathrm{Zr}_{45} \mathrm{Ti}_{5}$ alloy reflects the tuned criticality, showing a remarkably quantitative agreement with the slip statistics of dislocation motions in polycrystals. This dynamic critical behavior exhibits a novel unexplored approach for the deformation of the nanocrystal/glass phase composite, which is possibly valid for other composite systems that share similar dynamics, including the nano-glassy materials, and the oxidized glasses at the mesoscopic scale. 


\section{Acknowledgements}

The work described in this paper was supported by grants from the MOST (No. 2015CB856800), NSF of China (Nrs. 51171098, 51222102 and 11271339). Additional support through the German Science Foundation (DFG) under the Leibniz Program (grant EC 111/26-1) and the European Research Council under the ERC Advanced Grant INTELHYB (grant ERC-2013-ADG-340025) is gratefully acknowledged. PKL very much appreciates the financial support from the US National Science Foundation (CMMI-1100080) with C. V. Cooper as the contract monitor. KAD and PKL are very grateful for the support from the US Department of Energy (DOE), Office of Fossil Energy, National Energy Technology Laboratory (DE-FE-0008855, DE-FE-0011194, and DE-FE-0024054), and the US Army Research Office (W911NF-13-1-0438) with V. Cedro, J. Mullen, S. Markovich, R. Dunst, S. Mathaudhu, and D. Stepp as contract monitors.

\section{References}

Antonaglia, J., Wright, W.J., Gu, X.J., Byer, R.R., Hufnagel, T.C., LeBlanc, M., Uhl, J.T., Dahmen, K.A., 2014a. Bulk metallic glasses deform via slip avalanches. Phys. Rev. Lett. 112, 155501.

Antonaglia, J., Xie, X., Schwarz, G., Wraith, M., Qiao, J.W., Zhang, Y., Liaw, P.K., Uhl, J.T., Dahmen, K.A., 2014b. Tuned critical avalanche scaling in bulk metallic glasses. Sci. Rep. 4, 4382.

Bailey, N.P., Schiotz, J., Jacobsen, K.W., 2006. Atomistic simulation study of the shear-band deformation mechanism in Mg-Cu metallic glasses. Phys. Rev. B. 73, 064108.

Batra, R.C., Lear, M.H., 2005. Adiabatic shear banding in plane strain tensile deformations of 11 thermoelastoviscoplastic materials with finite thermal wave speed. Int. J. Plasticity 21, 1521-1545.

Ben-Zion, Y., Dahmen, K.A., Uhl, J.T., 2011. A unifying phase diagram for the dynamics of sheared solids and granular materials. Pure Appl. Geophys. 68, 2221-2237.

Bharathi, M. S., Lebyodkin, M., Ananthakrishna, G., Fressengeas, C., Lp., K., 2001. Multifractal burst in the spatiotemporal dynamics of jerky flow. Phys. Rev. Lett. 87, 165508.

Bulatov, V.V., Argon, A.S., 1994. A stochastic model for continuum elasto-plastic behavior. I. Numerical approach and strain localization. Model. Simul. Mater. Sci. Eng. 2, 167-184.

Chen, M.W., Inoue, A., Zhang, W., Sakurai, T., 2006. Extraordinary plasticity of ductile bulk metallic glasses. Phys. Rev. Lett. 96, 245502.

Chen, M.W., Ma, E., Hemker, K.J., Sheng, H.W., Wang, Y.M., Cheng, X.M., 2003. Deformation twinning in nanocrystalline aluminum. Science 300, 1275-1277. 
Chen, Y., Jiang, M.Q., Dai, L.H., 2013, Collective evolution dynamics of multiple shear bands in bulk metallic glasses. Int. J. Plasticity 50, 18-36.

Cheng, Y.Q., Han, Z., Li, Y., Ma, E., 2009. Cold versus hot shear banding in bulk metallic glass. Phys. Rev. B 80, 134115.

Csikor, F.F., Motz, C., Weygand, D., Zaiser, M., Zapperi, S., 2007. Dislocation avalanches, strain bursts, and the problem of plastic forming at the micrometer scale. Science 318, 251-254.

Cullity, B.D., 1959. Elements of X-ray diffraction. Addison-wesley publishing company, INC. Reading.

Dahmen, K.A. Ben-Zion, Y., Uhl, J.T., 2011. A simple analytic theory for the statistics of avalanches in sheared granular materials. Nat. Phys. 7, 554-557.

Dahmen, K.A., Ben-Zion, Y., Uhl, J.T., 2009. Micromechanical model for deformation in solids with universal predictions for stress-strain curves and slip avalanches. Phys. Rev. Lett. 102, 175501.

Dimiduk, D.M, Woodward, C., LeSar, R., Uchic, M.D., 2006. Scale-free intermittent flow in crystal plasticity. Science, 312, 1188-1190.

Ding, J., Patinet, S., Falk, M.L., Cheng, Y.Q., Ma, E., 2014. Soft spots and their structural signature in a metallic glass. Proc. Natio. Acad. Soc. USA 111, 14052-14056.

Eshelby, J. D., 1957. The determination of the elastic field of an ellipsoidal inclusion, and related problems. Proc. R. Soc. Lond. A 241, 376-396.

Fan, C., Li, H.Q., Kecskes, L.J., Tao, K.X., Choo, H., Liaw, P.K., Liu, C.T., 2006. Mechanical behavior of bulk amorphous alloys reinforced by ductile particles at cryogenic temperatures. Phys. Rev. Lett. 96, 145506.

Fornell, J., Concustell, A., Suriñach, S., Li, W.H., Cuadrado, N., Gebert, A., Baró, M.D., Sort, J., 2009. Yielding and intrinsic plasticity of Ti-Zr-Ni-Cu-Be bulk metallic glass. Int. J. Plasticity $25,1540-1559$.

Freltoft, T., Kjems, J.K., Sk., S., 1986. Power-law correlations and finite-size effects in silica particle aggregates studied by small-angle neutron scattering. Phys. Rev. B. Condens Matter. $33,269-275$.

Georgarakis, K., Aljerf, M., Li, Y., LeMoulec, A., Charlot, F., Yavari, A. R., Chornokhvostenko, K., Tabachnikova, E., Evangelakis, G. A., Miracle, D. B., Greer, A. L., Zhang, T., 2008. Shear band melting and serrated flow in metallic glasses. Appl. Phys. Lett. 93, 031907.

Gupta, H.S., Wagermaier, W., Zickler, G.A., Aroush, D.R-B., Funari, S.S., Roschger, P., Wagner, H.D., Fratzl, P., 2005. Nanoscale Deformation Mechanisms in Bone. Nano Lett. 5, 2108-2011. Hirth, J.P., Lothe J., 1982, New York: John Wiley \& Sons, INC., Theory of Dislocations (Sec. Ed.). 
Huang, Y., Khong, J.C., Connolley, T., Mi, J., 2014. The onset of plasticity of a Zr-based bulk metallic glass. Int. J. Plasticity 60, 87-100.

Jiang, W.H., Atzmon, M., 2003. Rate dependence of serrated flow in a metallic glass. J. Mater. Res. $18,755-757$.

Jiang, W.H., Fan, G.J., Liu, F.X., Wang, G.Y., Choo, H., Liaw, P.K., 2008. Spatiotemporally inhomogeneous plastic flow of a bulk-metallic glass. Int. J. Plasticity 24, 1-16.

Kirkwood, D.H. 1994. Semisolid metal processing. Int. Mater. Rev. 39, 173-189.

Klaumünzer, D., Lazarev, A., Maß, R., Dalla Torre, F.H., Vinogradov , A., Löffler, J.F., 2011 a. Probing shear-band initiation in metallic glasses. Phys. Rev. Lett. 107, 185502.

Klaumünzer, D., Maßß, R., Löffler, J.F., 2011 b. Stick-slip dynamics and recent insights into shear banding in metallic glasses. J. Mater. Res. 26, 1453-1463.

Koslowski M., LeSar R., Thomson R., 2004 Avalanches and scaling in plastic deformation. Phys. Rev. Lett. 93,125502.

Kumar, J., Ananthakrishna, G., 2011. Multiscale modeling approach to acoustic emission during plastic deformation. Phys. Rev. Lett. 106, 106001.

Lewandoski, J.J., Greer, A.L., 2006. Temperature rise at shear bands in metallic glasses. Nat. Mater. $5,15-18$.

Liu, Z.Y., Yang, Y., 2012. A mean-field model for anelastic deformation in metallic glasses. Intermetallics 26, 86-90.

Liu, Z.Y., Wang, G., Chan, K.C., Ren, J.L., Bian, X.L., Huang, Y.J., Xu, X.H., Zhang, D.S., Gao, Y.L., Zhai, Q.J., 2013a. Temperature dependent dynamics transition of intermittent plastic flow in a metallic glass. I. Experimental investigations. J. Appl. Phys. 114, 033520.

Liu, Z.Y., Wang, G., Chan, K.C., Ren, J.L., Bian, X.L., Huang, Y.J., Xu, X.H., Zhang, D.S., Gao, Y.L., Zhai, Q.J., 2013b. Temperature dependent dynamics transition of intermittent plastic flow in a metallic glass. II. Dynamics analysis. J. Appl. Phys. 114, 033521.

Lund, A.C., Schuh, C.A., 2007. Critical length scales for the deformation of amorphous metals containing nanocrystals. Philos. Mag. Lett. 87, 603-611.

McLelland, A.R.A., Henderson, N.G., Atkinson, H.V., Kirkwood, D.H., 1997. Anomalous rheological behaviour of semi-solid alloy slurries at low shear rates. Mater. Sci. Eng. A 232, 110-118.

Miller, R.E., Acharya, A., 2004. A stress-gradient based criterion for dislocation nucleation in crystals, J. Mech. Phys. Solids 52, 1507-1525.

Miracle, D.B., 2004. A structural model for metallic glasses. Nat. Mater. 3, 697-702.

Neuhaüser, S., 1993. Collective micro shear processes and plastic instabilities in crystalline and amorphous structures. Int. J. Plasticity 9, 421-435. 
Papanikolaou, S., Dimiduk, D.M., Choi, W.S., Sethna, J.P., Uchic, M.D., Woodward, C.F., Zapperi, S., 2012. Quasi-periodic events in crystal plasticity and the self-organized avalanche oscillator. Nature 490, 517-521.

Pauly, S., Gorantla, S., Wang, G., Kühn, U., Eckert, J., 2010a. Transformation-mediated ductility in CuZr-based bulk metallic glasses. Nat. Mater. 9, 473-477.

Pauly, S., Liu, G., Gorantla, S., Wang, G., Kühn, U., Eckert, J., 2010b. Criteria for tensile plasticity in $\mathrm{Cu}-\mathrm{Zr}-\mathrm{Al}$ bulk metallic glasses. Acta Mater. 58, 4883-4890.

Pauly, S., Liu, G., Wang, G., Kühn, U., Mattern, N., Eckert, J., 2009. Microstructural heterogeneities governing the deformation of $\mathrm{Cu}_{47.5} \mathrm{Zr}_{47.5} \mathrm{Al}_{5}$ bulk metallic glass composites. Acta Mater. 57, 5445-5453.

Peterlik, H., Roschger, P., Klaushofer, K., Fratzl, P., 2006. From brittle to ductile fracture of bone. Nat. Mater. 5, 52-55.

Qiao, J.W., Jia, H.L., Zhang, Y., Liaw, P.K., Li, L.F., 2012. Multi-step shear banding for bulk metallic glasses at ambient and cryogenic temperatures. Mater. Chem. Phys. 136, 75-79.

Ren, J.L., Chen, C., Liu, Z.Y., Li, R., Wang, G., 2012. Plastic dynamics transition between chaotic and self-organized critical states in a glassy metal via a multifractal intermediate. Phys. Rev. B $86,134303$.

Ren, J.L., Chen, C., Wang, G., Cheung, W.S., Sun, B.A., Mattern, N., Siegmund, S., Eckert, J., 2014. Various sizes of sliding event bursts in the plastic flow of metallic glasses based on a spatiotemporal dynamic model. J. Appl. Phys. 116, 033520.

Ren, J.L., Chen, C., Wang, G., Mattern, N., Eckert, J., 2011. Dynamics of serrated flow in a bulk metallic glass. AIP Advances 1, 032158.

Richeton, T., Weiss, J., Louchet, F., 2005. Breakdown of avalanche critical behaviour in polycrystalline plasticity. Nat. Mater. 4, 465-469.

Ritchie, R.O., 2011. The conflicts between strength and toughness. Nat. Mater. 10, 817-822.

Sarmah, R., Ananthakrishna, G., Sun, B.A., Wang, W.H., 2011. Hidden order in serratfdw of metallic glasses. Acta Mater. 59, 4482-4493.

Schuh, C.A., Lund, A.C., 2009. Atomistic basis for the plastic yield criterion of metallic glass. Nat. Mater. 2, 449-452.

Schuh, C.A., Lund, A.C., Nieh, T.G., 2004. New regime of homogeneous flow in the deformation map of metallic glasses: elevated temperature nanoindentation experiments and mechanistic modeling. Acta Mater. 52, 5879-5891.

See supplemental materials for additional information on the technical details.

Smith, W., Hashemi, J., 2006. Foundations of Materials Science and Engineering. McGraw-Hill InterNatureional Edition, 4th ed. 
Song, S.X., Nieh, T.G., 2011. Direct measurements of shear band propagation in metallic glasses. Intermetallics 19, 1968-1977.

Song, S.X., Nieh, T.G., 2009. Flow serration and shear-band viscosity during inhomogeneous deformation of a Zr-based bulk metallic glass. Intermetallics 17, 762-767.

Sun, B.A., Liu, Z.Y., Yang, Y., Liu, C.T., 2014. Delayed shear banding and evolution of local plastic flow in a metallic glass. Appl. Phys. Lett. 105, 091904.

Sun, B.A., Pauly, S., Tan, J., Stoica, M., Wang, W.H., Kühn, U., Eckert, J., 2012. Serrated flow and stick-slip deformation dynamics in the presence of shear-band interactions for a Zr-based metallic glass. Acta Mater. 60, 4160-4171.

Sun, B.A., Yu, H.B., Jiao, W., Bai, H.Y., Zhao, D.Q., Wang, W.H., 2010. Plasticity of ductile metallic glasses: a self-organized critical state. Phys. Rev. Lett. 105, 035501.

Trexler, M.M., Thadhani, N.N., 2010. Mechanical properties of bulk metallic glasses. Prog. Mater. Sci. 55, 759-839.

Wang, G., Chan, K.C., Xia, L., Yu, P., Shen, J., Wang, W.H., 2009. Self-organized intermittent plastic flow in bulk metallic glasses. Acta Mater. 57, 6146-6155.

Wang, G., Liu, Y.H., Yu, P., Zhao, D.Q., Pan, M.X., Wang, W.H., 2006. Structural evolution in TiCu-based bulk metallic glass with large compressive plasticity. Appl. Phys. Lett. 89, 251909.

Wang, G., Pauly, S., Gorantla, S., Mattern, N., Eckert, J., 2014. Plastic flow of a $\mathrm{Cu}_{50} \mathrm{Zr}_{45} \mathrm{Ti}_{5}$ bulk metallic glass composite. J. Mater. Sci. Technol. 30, 609-615.

Wang, S.G., Xu, J., 2013. Strengthening and toughening of Mg-based bulk metallic glass via in-situ formed B2-type AgMg phase. J. Non Cryst. Solids 379, 40-47.

Wu, F.F., Zheng, W., Wu, S.D., Zhang, Z.F., Shen, J., 2011a. Shear stability of metallic glasses. Int. J. Plasticity 27, 560-575.

Wu, Y., Bei, H., Wang, Y.L., Lu, Z.P., George, E.P., Gao, Y.F., 2015. Deformation-induced spatiotemporal fluctuation, evolution and localization of strain fields in a bulk metallic glass. Int. J. Plasticity 71, 136-145.

Wu, Y., Wang, H., Wu, H.H., Zhang, Z.Y., Hui, X.D., Chen, G.L., Ma, D., Wang, X.L., Lu, Z.P., 2011b. Formation of $\mathrm{Cu}-\mathrm{Zr}-\mathrm{Al}$ bulk metallic glass composites with improved tensile properties. Acta. Mater. 59, 2928-2936.

Wu, Y., Xiao, Y.H., Chen, G.L., Liu, C.T., Lu, Z.P., 2010. Bulk metallic glass composites with transformation-mediated work-hardening and ductility. Adv. Mater. 22, 2770-2773.

$\mathrm{Xu}$, J., Ma, E., 2014. Damage-tolerant Zr-Cu-Al-based bulk metallic glasses with record-breaking fracture toughness. J. Mater. Res. 29, 1489-1499. 
Yang, B., Morrison, M.L., Liaw, P.K., Buchanan, R.A., Wang, G.Y., Liu, C.T., Denda, M., 2005. Dynamic evolution of nanoscale shear bands in a bulk-metallic glass. Appl. Phys. Lett. 86, 141904.

Ye, J.C., Lu, J., Liu, C.T., Wang, Q., Yang, Y., 2010. Atomistic free-volume zones and inelastic deformation of metallic glasses. Nat. Mater. 9, 619-623.

Zaiser, M., Moretti, P., 2005. Fluctuation phenomena in crystal plasticity-a continuum model. J. Stat. Mech. 08, 1748-5468, P08004.

Zhang, Y., Greer, A.L., 2006. Thickness of shear bands in metallic glasses. Appl. Phys. Lett. 89, 071907.

Zhao, P., Li, J., Wang, Y., 2013. Heterogeneously randomized STZ model of metallic glasses: Softening and extreme value statistics during deformation. Int. J. Plasticity 40, 1-22. 
Table I Mechanical properties of four alloys.

\begin{tabular}{|c|c|c|c|c|}
\hline Sample ID & PT I & PT II & PT III & PT IV \\
\hline Elastic modulus $(\mathrm{GPa})$ & $81 \pm 5$ & $79 \pm 6$ & $74 \pm 6$ & $62 \pm 6$ \\
\hline Yielding strength $(\mathrm{MPa})$ & $1,740 \pm 47$ & $1,708 \pm 17$ & $1,618 \pm 99$ & $1,589 \pm 123$ \\
\hline Maximum strength $(\mathrm{MPa})$ & $1,951 \pm 47$ & $1,855 \pm 67$ & $1,974 \pm 59$ & $2,034 \pm 54$ \\
\hline Maximum strain & $0.044 \pm 0.002$ & $0.048 \pm 0.005$ & $0.070 \pm 0.004$ & $0.106 \pm 0.003$ \\
\hline
\end{tabular}

Table II Microstructure parameters of four alloys.

\begin{tabular}{|c|c|c|c|c|}
\hline Sample ID & PT I & PT II & PT III & PT IV \\
\hline$D_{a v r}(\mathrm{~nm})$ & - & $8.3 \pm 0.9$ & $11.1 \pm 1.8$ & $20.2 \pm 2.8$ \\
\hline$f_{c}(\%)$ & 0 & $7.8 \pm 1.0$ & $16.8 \pm 1.9$ & $35.0 \pm 2.9$ \\
\hline$L(\mathrm{~nm})$ & $\infty$ & 15.7 & 13.0 & 21.6 \\
\hline$s(\mathrm{~nm})$ & $\geq 10$ & $7.5 \pm 1.1$ & $4.3 \pm 0.8$ & $2.4 \pm 0.8$ \\
\hline
\end{tabular}




\section{Figure captions}

Fig. 1. Compressive true stress-strain curves of four $\mathrm{Cu}_{50} \mathrm{Zr}_{45} \mathrm{Ti}_{5}$ alloys with different structures. The inset shows the XRD patterns of these four alloys. The dash-point rectangle covers the large serration events in the plastic regime of PT IV.

Fig. 2. Fractographies of four fractured $\mathrm{Cu}_{50} \mathrm{Zr}_{45} \mathrm{Ti}_{5}$ alloys. (a), (c), (e), and (g) present the fracture-surface morphologies of PT I, PT II, PT III, and PT IV, respectively. (b), (d), (f) and (h) present the lateral surface morphologies of PT I, PT II, PT III, and PT IV, respectively. Blue dot arrows point to the shear bands.

Fig. 3. Microstructures of four fractured samples observed by TEM. (a), (c), (e), and (g) present the high-resolution TEM images of PT I, PT II, PT III, and PT IV, respectively. White circles in (c) and (e) point to the nanocrystals. (b), (d), (f), and (h) present the dark-field images of PT I, PT II, PT III, and PT IV, respectively. Insets show the selected area electron diffraction patterns.

Fig. 4. Enlarged stress-strain curves in the plastic regime of four $\mathrm{Cu}_{50} \mathrm{Zr}_{45} \mathrm{Ti}_{5}$ alloys.

Fig. 5. Elastic-energy density stored in the serration events of four $\mathrm{Cu}_{50} \mathrm{Zr}_{45} \mathrm{Ti}_{5}$ alloys as a function of plastic strain.

Fig. 6. Cumulative probability distributions of strain-burst sizes. Open scattering points represent the experimental results measured from the compression tests. Solid lines are fitting curves by Eq. (2).

Fig. 7. Shear stress and shear-strain rate in each serration event of four $\mathrm{Cu}_{50} \mathrm{Zr}_{45} \mathrm{Ti}_{5}$ alloys. (a) Shear stress measured from the serration event as a function of plastic strain. (b) Calculated shear-strain rate as a function of plastic strain. The averaged shear-strain rates are listed. (c) The shear viscosity versus the true strain of four samples, respectively. The average shear viscosity is listed.

Fig. 8. Sketch of the shear-banding behavior in the $\mathrm{Cu}_{50} \mathrm{Zr}_{45} \mathrm{Ti}_{5}$ alloy. (a) Sketch of the shear deformation. $\gamma$ is the shear strain, $\varepsilon_{n}$ is the normal strain, $\tau$ is the shear stress, $\theta$ is the fracture angle, and $\Delta \varepsilon$ is the plastic strain generated from each serration events. (b) Shear-banding behavior in PT I. Nanocrystals contained in shear bands, and shear-band slipping across the glassy phase can be 
observed. (c) Shear-banding behavior in PT III. Large nanocrystals can prohibit shear banding. (d) Shear-banding behavior in PT IV. Twinning can cooperate with shear banding.

Fig. 9. The $\Delta \delta_{\mathrm{c}}$ value as a function of shear viscosity.

Fig. 10. The $\Delta \delta_{\mathrm{c}}$ value as a function of the $s$ value, which shows a power-law relationship. 


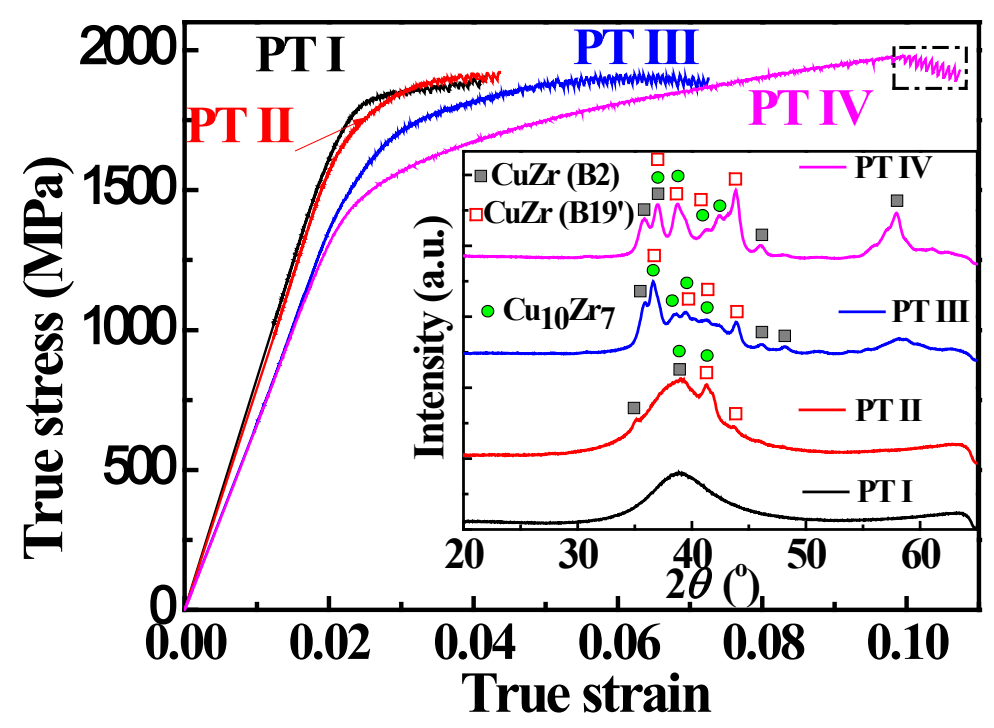

Fig. 1. Tong, et al. 

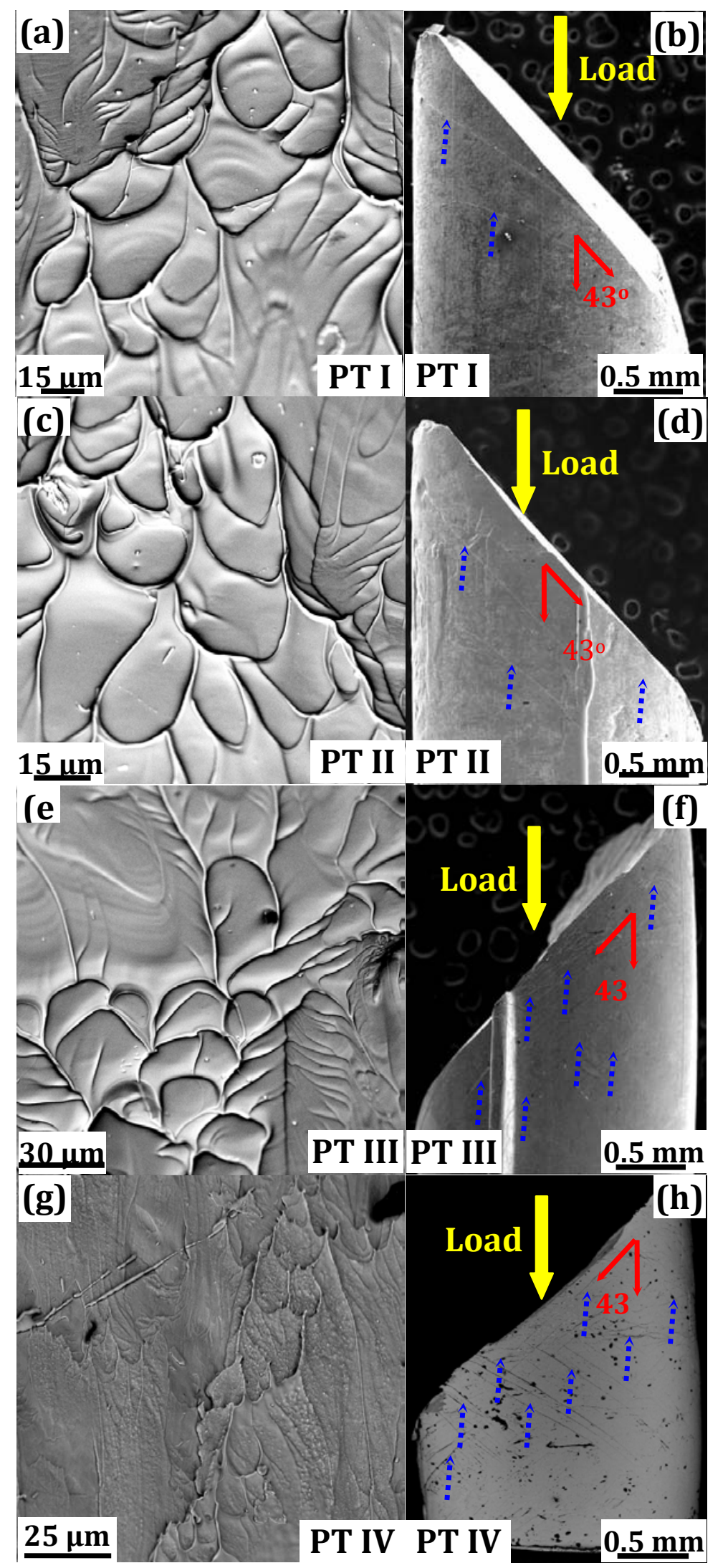

Fig. 2. Tong, et al. 


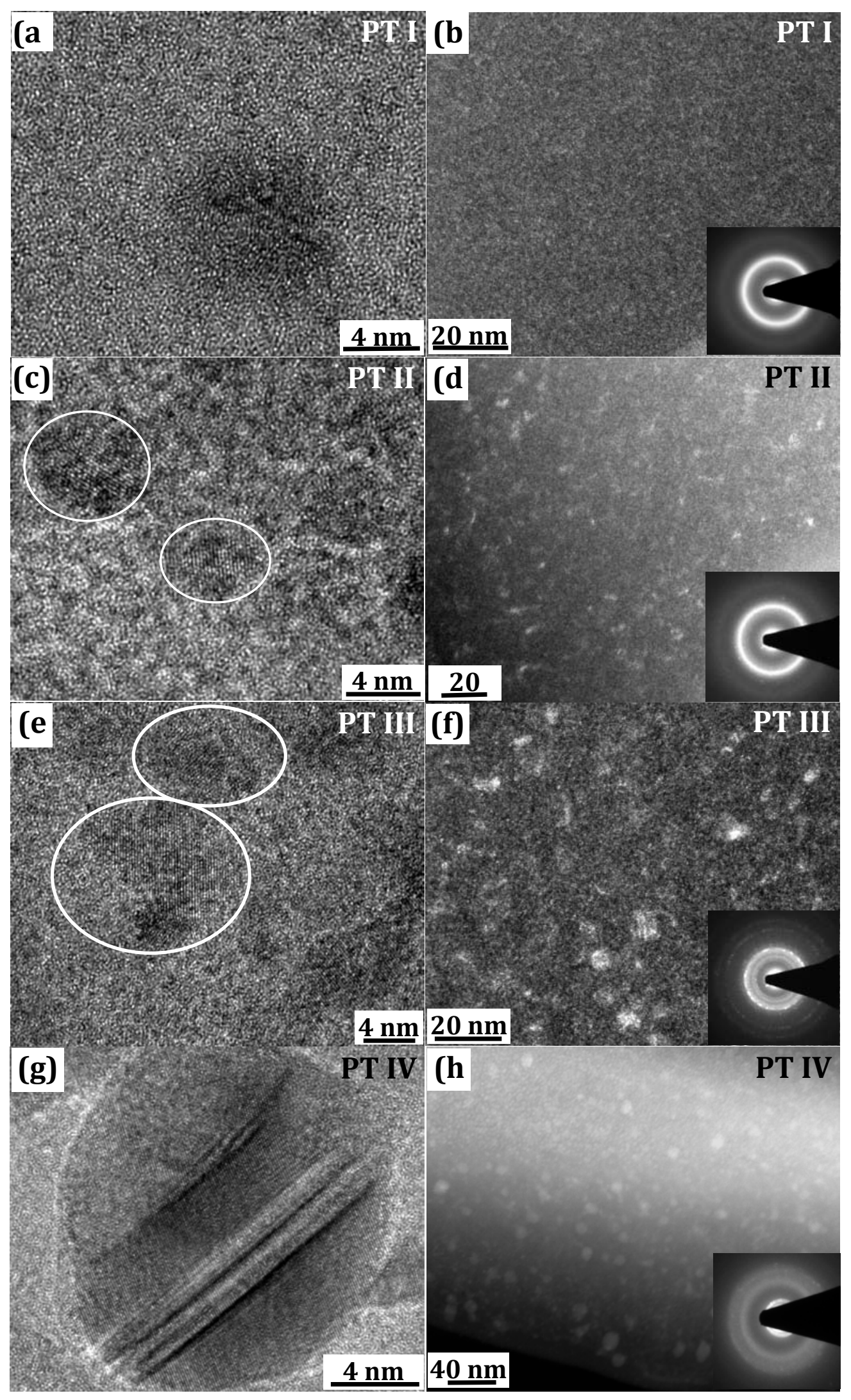

Fig. 3. Tong, et al.

(b) $800-v_{C Z T I}=45.27 \mathrm{MPa} / \mathrm{s}$ 


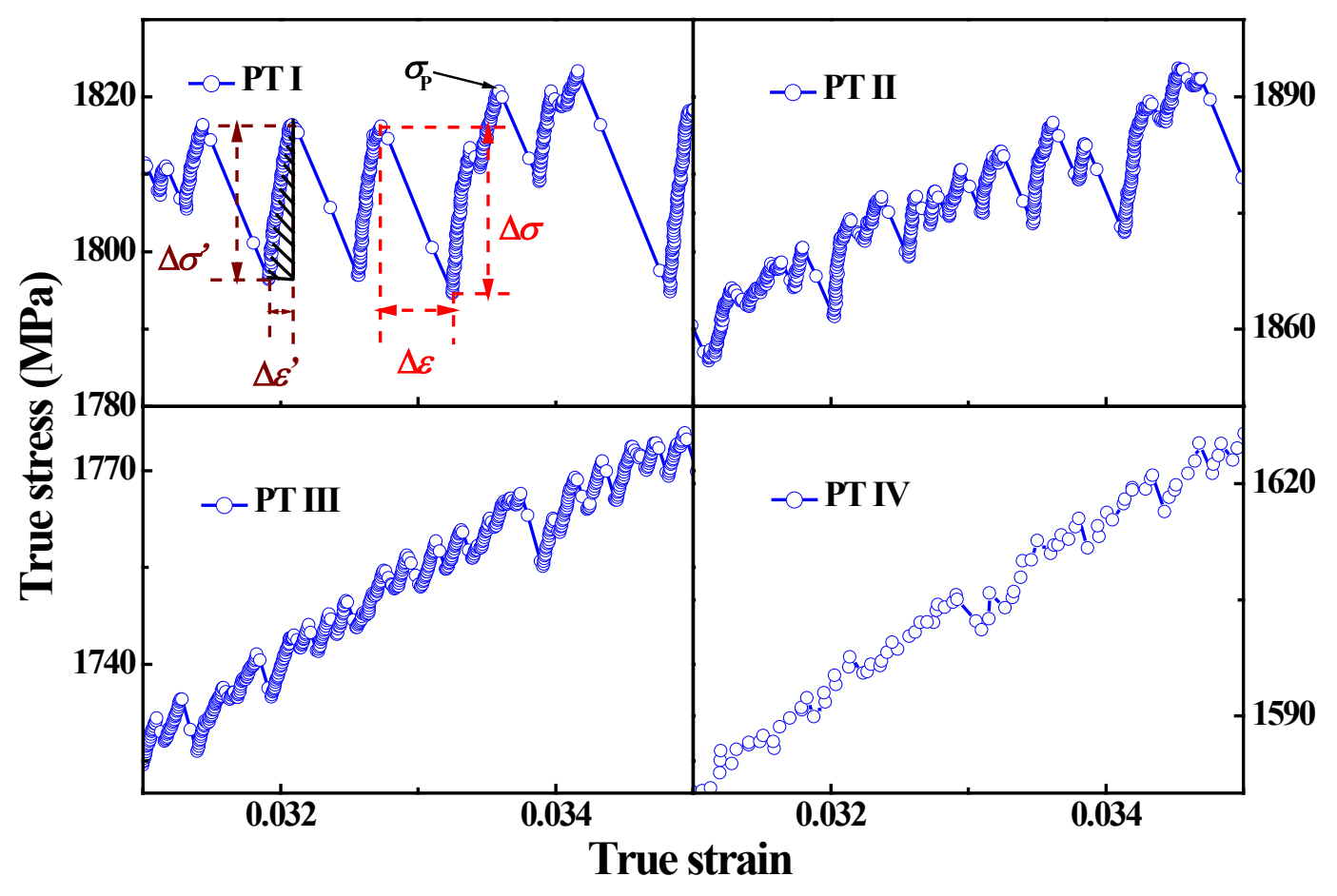

Fig. 4. Tong, et al. 


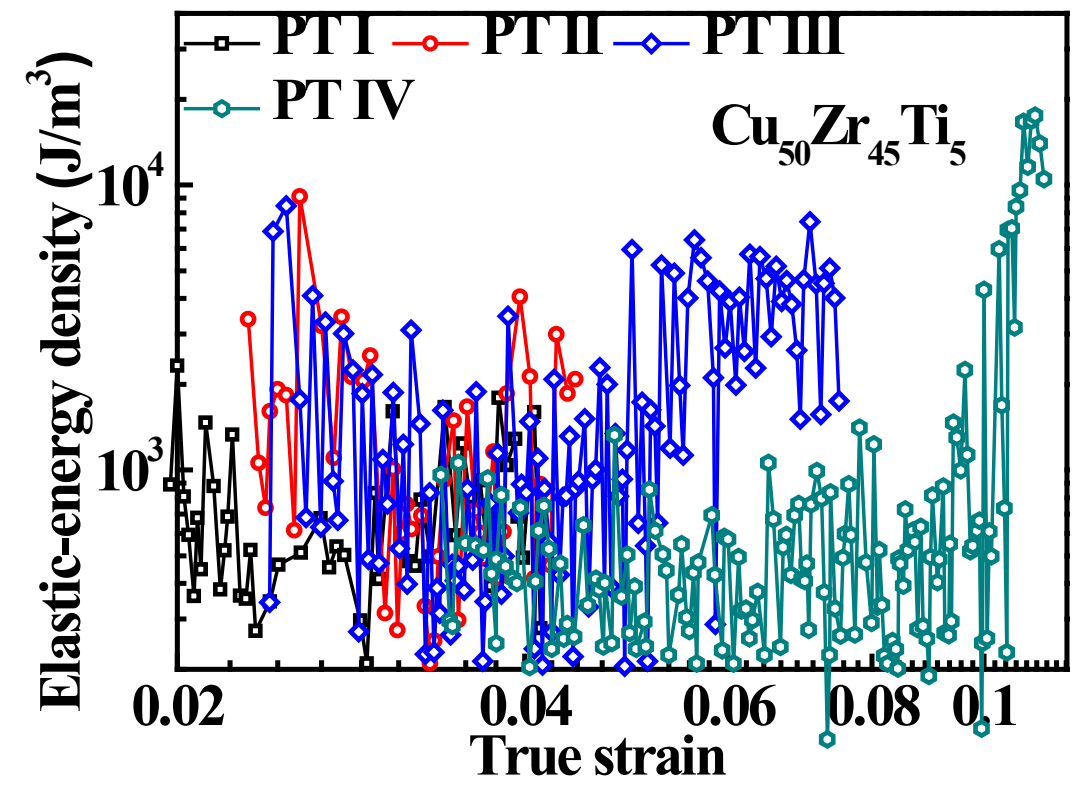

Fig. 5. Tong, et al.

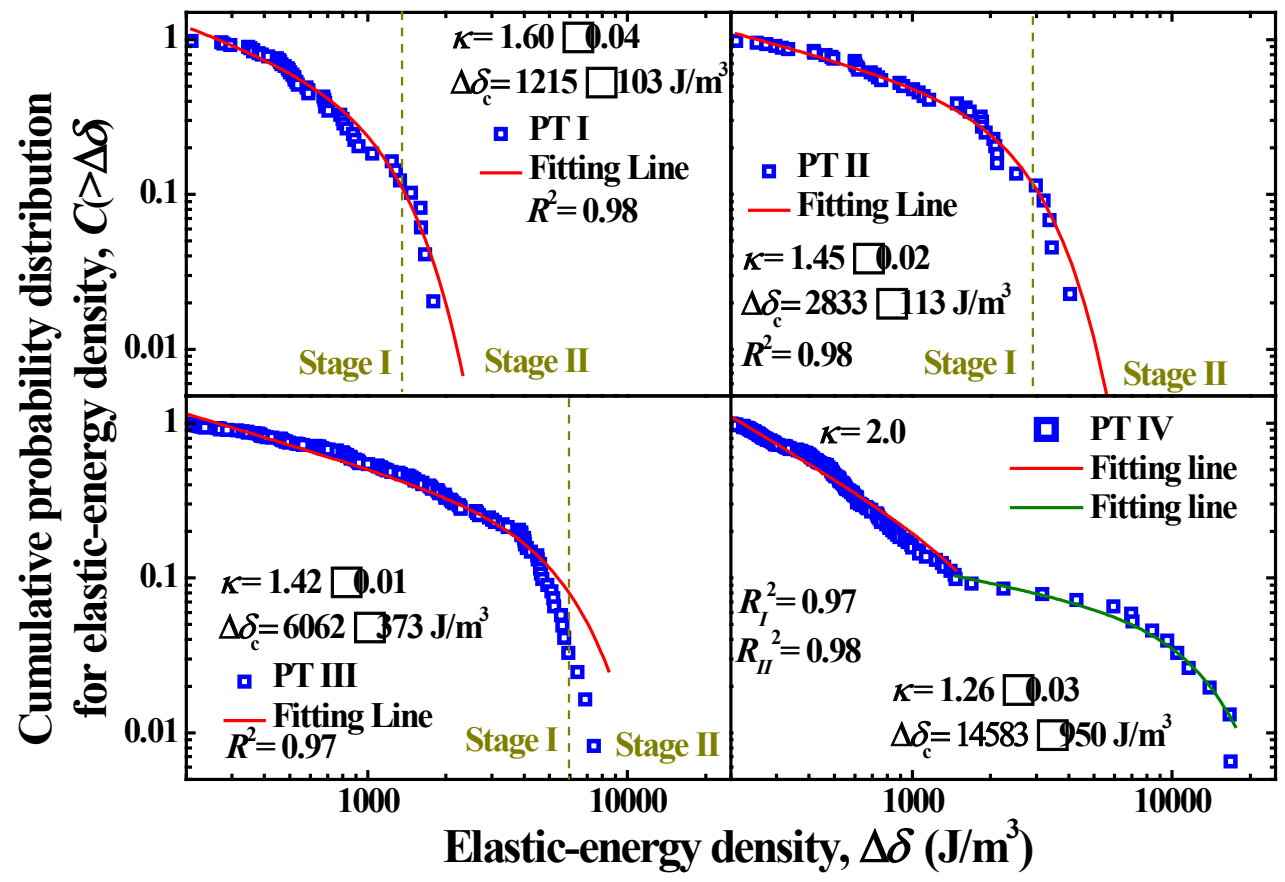

Fig. 6. Tong, et al. 

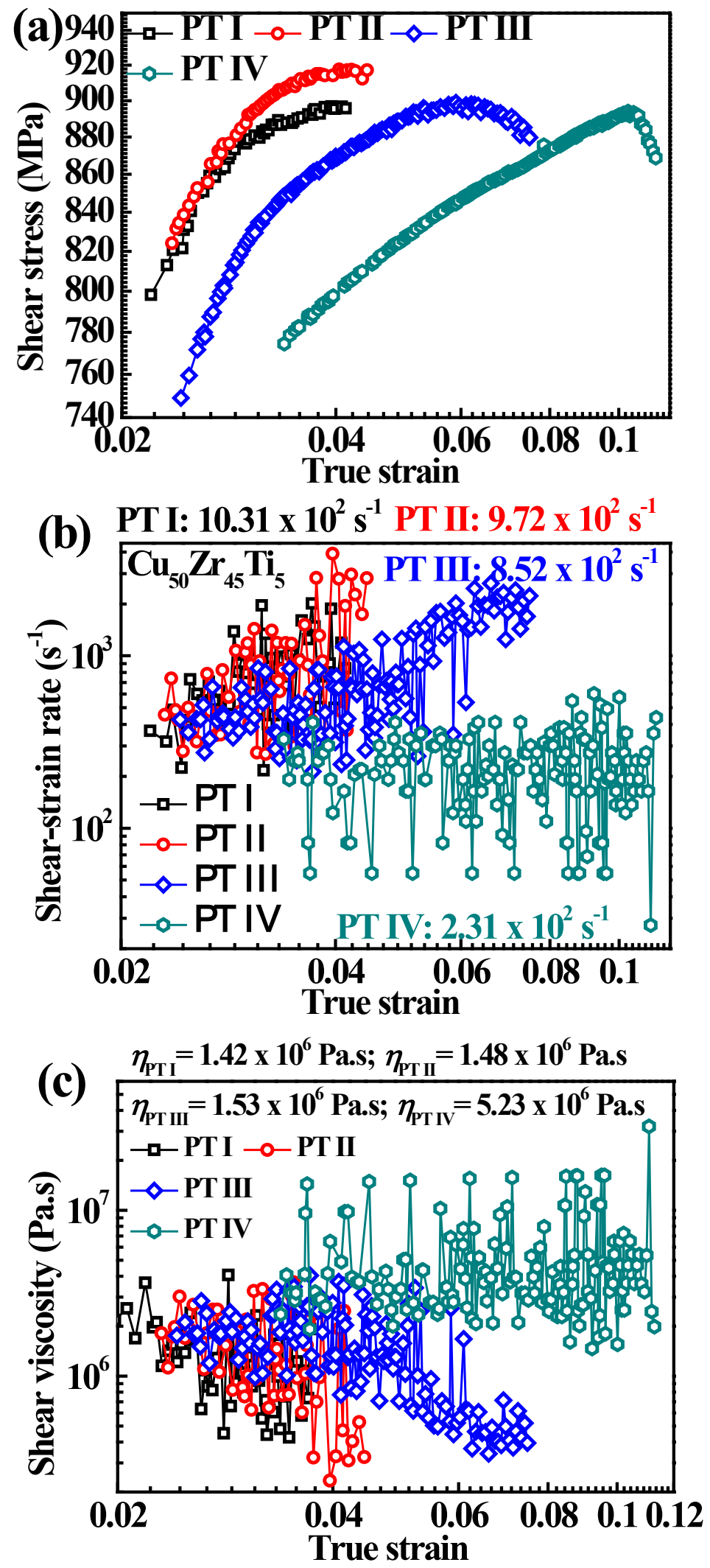

Fig. 7. Tong, et al. 




Fig. 8. Tong, et al. 


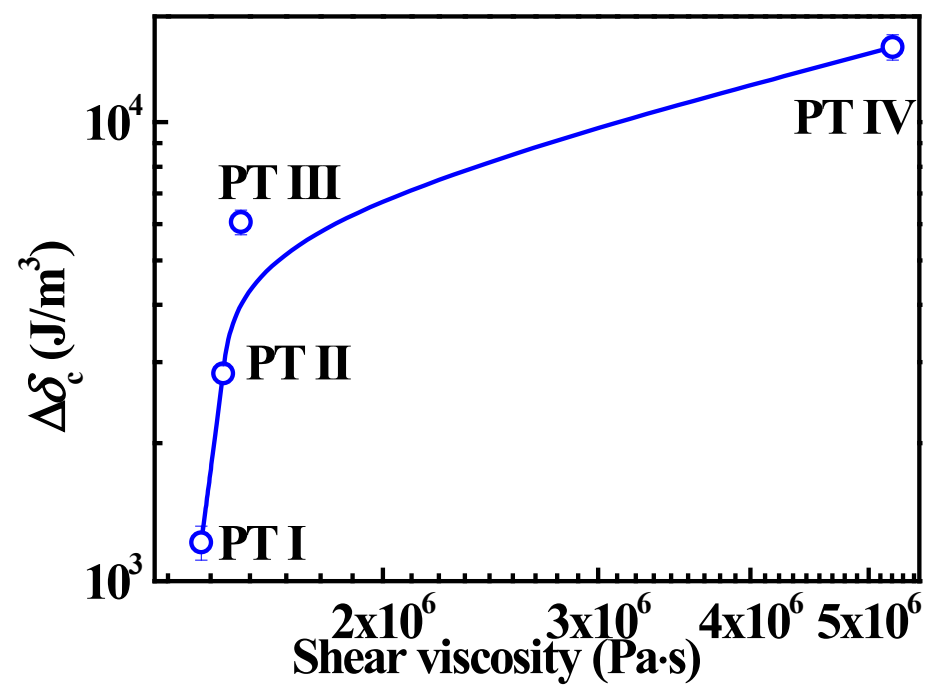

Fig. 9. Tong, et al. 


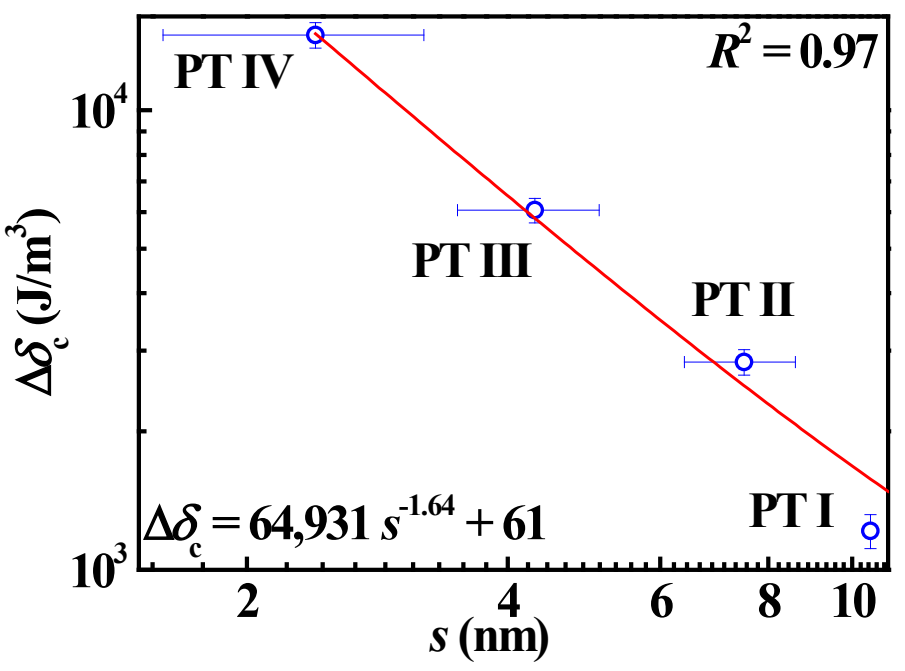

Fig. 10. Tong, et al. 